\title{
Aspirin Administration Affects Neurochemical Characterization of Substance P-Like Immunoreactive (SP-LI) Nodose Ganglia Neurons Supplying the Porcine Stomach
}

\author{
Liliana Rytel $\mathbb{D}^{1}$ and Jarosław Całka $\mathbb{D}^{2}$ \\ ${ }^{1}$ Department of Internal Disease with Clinic, Faculty of Veterinary Medicine, University of Warmia and Mazury, Poland \\ ${ }^{2}$ Department of Clinical Physiology, Faculty of Veterinary Medicine, University of Warmia and Mazury, Poland \\ Correspondence should be addressed to Liliana Rytel; liliana_rytel@wp.pl
}

Received 15 September 2019; Revised 9 April 2020; Accepted 18 May 2020; Published 13 June 2020

Academic Editor: Ali A. Khraibi

Copyright (c) 2020 Liliana Rytel and Jarosław Całka. This is an open access article distributed under the Creative Commons Attribution License, which permits unrestricted use, distribution, and reproduction in any medium, provided the original work is properly cited.

\begin{abstract}
Background. Acetylsalicylic acid (ASA) is a commonly used anti-inflammatory, antipyretic, and analgesic drug, which has many side effects on the gastric mucosal layer. Despite this, knowledge concerning the influence of ASA on neuronal cells supplying the stomach is very scanty. Methods. This investigation was performed on ten immature gilts of the Large White Polish race divided into two groups (five animals in each): a control group and animals which were treated with ASA. The retrograde neuronal tracer Fast Blue (FB) was injected into the prepyloric region of the stomach in all animals. ASA was then given orally to the experimental (ASA) group of gilts from the seventh day after FB injection to the 27th day of the experiment. After this period, all animals were euthanized. Immediately after euthanasia, nodose ganglia (NG) were collected and subjected to a standard double-labelling immunofluorescence technique using antibodies directed toward substance P (SP) and other selected neuronal factors, such as galanin (GAL), neuronal isoform of nitric oxide synthase (nNOS), vasoactive intestinal polypeptide (VIP), and calcitonin gene-related peptide (CGRP). Key Results. The obtained results show that SP-LI neurons located in NG supplying the porcine stomach were also immunoreactive to all the above-mentioned neuronal factors. Moreover, ASA administration caused an increase in the degree of colocalization of SP with other neuronal active substances, and the most visible changes concerned the number of neurons simultaneously immunoreactive to SP and CGRP. Conclusions and Inferences. These observations indicate that the population of SP-LI neurons supplying the stomach is not homogeneous and may undergo changes after ASA administration. These changes are probably connected with inflammatory processes and/or neuroprotective reactions although their exact mechanisms remain unknown.
\end{abstract}

\section{Introduction}

The innervation of the gastrointestinal (GI) tract consists of two main components. The first of them is the enteric nervous system (ENS) located in the wall of the digestive tract and built from the large number of neurons grouped in the enteric ganglia interconnected with a dense network of fibers and forming intramural ganglionated plexuses [1, $2]$. The morphology and number of these plexuses depend on animal species and the segment of the digestive tract [3-6]. Two types of enteric ganglia can be identified in the stomach. The first of them is myenteric ganglia which, together with nerves connecting the particular ganglia, form a clearly visible myenteric plexus located between the longitudinal and circular muscle layers [7]. The other component of the gastric ENS is submucosal ganglia positioned near the lamina propria of the mucosal layer which, contrary to myenteric ganglia, do not form a plexus [7]. The second component of the gastrointestinal innervation is the extrinsic innervation. Neurons supplying the GI tract and controlling the activity of the ENS are localized (in accordance with their functions) in vagal ganglia, prevertebral sympathetic ganglia, and dorsal root ganglia $[8-10]$. 
It should be noted that both enteric neurons and cells participating in extrinsic intestinal innervation vary widely in terms of neurochemical characterization. To date, several dozen active substances have been noted in neuronal cells supplying the GI tract $[11,12]$. One of the more important active substances occurring in neuronal cells supplying the gastrointestinal tract is substance $\mathrm{P}$ (SP) [13].

$\mathrm{SP}$ is an undecapeptide which, together with neurokinins $A$ and $B$, as well as neuropeptides $K$ and $\gamma$, belongs to the tachykinin neuropeptide family [14]. It was described for the first time in the 1930s [15], and since then, it has been observed in both intrinsic and extrinsic intestinal innervations in numerous mammal species, including humans $[11,16-18]$. SP may act via three types of $G$ proteincoupled NK receptors (NK1, NK2, and NK3), but the highest affinity is exhibited toward the NK1receptor. Previous studies showed that the exact functions of SP in the digestive tract depend on the intestinal fragment, animal species, and type of activated receptor [19, 20].

Multidirectional functions of SP in the regulation of the gastrointestinal activity have been described in previous studies. It is known that this substance takes part in the regulation of the smooth muscle contractility and the effects of this activity differ depending on the type of activated receptor. An SP-induced increase in the intestinal muscle contractility has been observed after excitation of the NK3 receptor, while the stimulation of the NK1 receptor causes relaxatory effects [21]. SP may also modulate the intestinal immunological system. This activity involves the activation of NK1 receptors located on lymphocyte and macrophage surfaces and an increase in the secretion of proinflammatory factors $[22,23]$. Substance $\mathrm{P}$ is also involved in regulatory processes connected with intestinal secretory functions and mesenteric blood flow [24]. But the most important functions of the SP in the gastrointestinal innervation seem to be the participation in sensory and pain stimulus conduction [25]. Previous studies also reported that levels of substance $\mathrm{P}$ in neurons supplying the GI tract can undergo significant changes under various pathological factors, such as inflammatory processes, nerve damage, and other intestinal and extraintestinal diseases $[11,13]$.

It should be noted that, contrary to the enteric nervous system, which is located in the wall of the digestive tract where the distribution and functions of SP are relatively well established [26], knowledge concerning this peptide within the extrinsic gastrointestinal innervation is rather scarce. In particular, little is known about the neurochemical characterization of SP-positive neurons and changes in colocalization of SP with other active substances under pathological factors.

Thus, the present study was aimed at investigating the influence of acetylsalicylic acid (ASA) administration on the chemical coding of SP-like immunoreactive (SP-LI) neurons located in the nodose ganglia and supplying the porcine stomach. ASA (also known as aspirin) is widely used as antiinflammatory, antipyretic, and analgesic medicine, which causes the inactivation of the cyclooxygenase (COX) enzyme [27]. Apart from therapeutic activity, aspirin also shows side effects which are particularly evident within the stomach, where taking this drug may result in the gastric mucosal injury [28]. It should be underlined that although the mechanisms of aspirin activity have been the subject of many studies [29], knowledge concerning its influence on extrinsic gastrointestinal innervation is extremely scarce [30]. On the other hand, it is relatively well established that aspirin may strongly affect the gastric mucosal layer, leading to its erosion and the breaking of the gastric mucosal barrier. These detrimental effects are based on the influence of ASA on the levels of adenosine triphosphate in mucosal cells, intensification of sodium transport, and changes in mucus composition, leading to injury of the gastric mucosal layer and the formation of gastric ulcers. Development of the local pathology evokes transduction of painful stimulation through the vagal sensory pathways that might affect the chemical plasticity of the nodose ganglion perikarya. Therefore, the objective of the current study was to analyze the effect of aspirin-induced stomach pathology on coexpression of galanin (GAL), neuronal isoform of nitric oxide synthase (nNOS), vasoactive intestinal polypeptide (VIP), and calcitonin gene-related peptide (CGRP) with substance P (SP) in nodose ganglion sensory perikarya [11].

\section{Materials and Methods}

2.1. Animals and Experimental Procedures. This investigation was performed on ten immature porcine of the Large White Polish breed (approximately 8 weeks old, about $20 \mathrm{~kg}$ b.w.), which were kept during the experiment in standard conditions suitable for the species and age of animals. All procedures during the study were performed in accordance with the instructions of the Local Ethical Committee in Olsztyn (Poland) (decision number 05/2010).

After a one-week adaptive period, the animals underwent general anesthesia with azaperone (Stresnil, Janssen Pharmaceutica N.V., Belgium; $4 \mathrm{mg} / \mathrm{kg}$ of body weight, i.m.) and sodium thiopental (Thiopental, Sandoz, Kundl-Rakusko, Austria; $10 \mathrm{mg} / \mathrm{kg}$ of b.w., i.v.) and gastroscopy examination (using the video endoscope Olympus GIF 145 with working length $1030 \mathrm{~mm}$ and diameter $9.8 \mathrm{~mm}$ ) to evaluate the gastric mucosal layer. A median laparotomy was then conducted, and the prepyloric region of the anterior stomach wall (the diamond-shaped area of the approximate size $4 \mathrm{~cm} \times 4 \mathrm{~cm}$, located about $3 \mathrm{~cm}$ before the gastric pylorus) was injected with $50 \mu \mathrm{l}$ of a $5 \%$ aqueous solution of Fast Blue (FB, Dr. K. Illing $\mathrm{GmbH} \& \mathrm{KG}$, Germany; ten injections, $1 \mu \mathrm{l}$ each) using a Hamilton syringe equipped with a 26 -gauge needle. Great attention was paid to avoiding any contamination of the surrounding tissues with $\mathrm{FB}$ due to the hydrostatic leakage from the injection canal.

After the surgery, pigs were randomly divided into two groups of five animals in each: a control group (C group) and an ASA group, in which aspirin (Bayer; $100 \mathrm{mg} / \mathrm{kg}$ b.w.) was administered. Aspirin was given orally once a day, $1 \mathrm{~h}$ before the morning feeding from the seventh day after surgery to the $27^{\text {th }}$ day of the experiment. After this period (on the $28^{\text {th }}$ day of the experiment), the pigs were subjected to general anesthesia, euthanized with an overdose of sodium thiopental (Thiopental, Sandoz, Kundl, Austria; 
TABle 1: Description of antibodies.

\begin{tabular}{lcccc}
\hline Antigen & Species of origin & Code & Dilution & \\
\hline Primary antibodies & & & & Supplier \\
GAL & Rabbit & AB2233 & $1: 2000$ & Millipore \\
nNOS & Rabbit & AB5380 & $1: 4000$ & Chemicon \\
VIP & Rabbit & VA 1285 & $1: 4000$ & BioGene \\
SP & Rat & $8450-0505$ & $1: 150$ & AbD Serotec \\
CGRP & Rabbit & AB5920 & & AbD Serotec \\
Secondary antibodies & & A10040 & $1: 1000$ & Invitrogen \\
Alexa Fluor 546 & Donkey anti-rabbit & A21208 & $1: 1000$ & Invitrogen \\
Alexa Fluor 488 & Donkey anti-rat & &
\end{tabular}

$20 \mathrm{mg} / \mathrm{kg}$ of body weight given intravenously), and perfused transcardially with $4 \%$ buffered paraformaldehyde ( $\mathrm{pH} 7.4$ ).

2.2. Tissue Collection. Right and left nodose ganglia (NG) were collected from all animals. Tissues were postfixed in 4\% paraformaldehyde for $20 \mathrm{~min}$, rinsed in $\mathrm{M}$ buffer solution (0.1 M, pH 7.4) for 72 hours, put into $18 \%$ sucrose solution, and stored at $4^{\circ} \mathrm{C}$ at least for three weeks. After this period, nodose ganglia were frozen at $-22^{\circ} \mathrm{C}$ and cut using a microtome (Microm HM-525, Germany) into $12 \mu \mathrm{m}$ thick sections, which were evaluated under fluorescence Olympus BX51 for the presence of FB-positive neuronal cells.

2.3. Double Immunofluorescence Technique. Sections with FB-positive neurons were subjected to the routine doublelabelling immunofluorescence method described previously by Rytel and Całka [11] with a mixture of two primary antibodies. One of them was an antibody directed toward substance $\mathrm{P}$ (rat, 1:150, AbD Serotec), and the second was an antibody directed toward one of the other neuronal factors, such as a calcitonin gene-related peptide (CGRP, rabbit, 1:4000, AbD Serotec), neuronal isoform of nitric oxide synthase (nNOS, used here as the marker of nitrergic neurons, rabbit, $1: 4000$, Chemicon), galanin (GAL, rabbit, $1: 2000$, Millipore), and vasoactive intestinal polypeptide (VIP, rabbit, 1:4000, BioGene). Complexes "primary antibody-appropriate antigen" were visualized by speciesspecific secondary antibodies conjugated with Alexa Fluor (Alexa Fluor 488 donkey anti-rat IgG and Alexa Fluor 546 donkey anti-rabbit IgG, Invitrogen, Carlsbad, CA, USA, working dilution $1: 1000$ ) (Table 1).

Standard control procedures of antibody specificity, including preabsorption, as well as "omission" and "replacement" tests, eliminated specific staining.

2.4. Evaluation of the Percentage of Neuronal Cells. Quantitative analysis was conducted using a fluorescence Olympus BX51 microscope with sets of filters for fluorochromes used in the present study. Microphotographs were taken using Cell-F image analysis software (Olympus, Tokyo, Japan). For evaluation of the degree of colocalization of SP with other neuronal factors within neurons supplying the stomach, at least $70 \mathrm{FB}+/ \mathrm{SP}+$ cell bodies in each nodose ganglion (left or right) were identified and examined for immunoreactivity to the particular substances investigated, and the number of $\mathrm{FB}+/ \mathrm{SP}+$ neurons was treated as $100 \%$. For example, unilateral identification in 85 animal $\mathrm{FB}+/ \mathrm{SP}+$ cells was treated as $100 \%$ and was then performed (e.g., 22 of $\mathrm{FB}+/ \mathrm{SP}+/ \mathrm{VIP}+$ neurons constituted 25.88\%). Data obtained in animals of one group were pooled and presented as mean \pm SEM.

The relatively low number of $\mathrm{FB}+/ \mathrm{SP}+$ cells evaluated for the presence of the particular neuronal factors was caused by the fact that the population of SP-positive cells supplying the stomach located in the nodose ganglia is not numerous [31].

2.5. Statistical Analysis. Statistical analyses were performed using GraphPad Prism 5 software (GraphPad Software, USA) and an ANOVA test with Bonferroni's multiple comparison post hoc test. The differences were considered statistically significant at $P \leq 0.05$.

2.6. Histopathological Examination. Moreover, routine histopathological staining with the application of the hematoxyli$\mathrm{n}$ /eosin method was performed on fragments of the gastric wall from the prepyloric region to evaluate histopathological changes after ASA administration.

\section{Results}

During the present investigation, all neuronal active substances studied were observed in SP-positive neurons located in nodose ganglia and supplying the prepyloric region of the stomach, both under physiological conditions and after ASA administration.

In control animals, the degree of colocalization of SP with other substances depended on the type of substance and, to a lesser extent, on the side of nodose ganglion localization (Table 2) (Figures 1 and 2; Figures 3 and 4). The highest number of the investigated neurons contained CGRP, which was found within $53.72 \% \pm 2.84 \%$ and $50.78 \% \pm 2.92 \%$ of all $\mathrm{FB}+/ \mathrm{SP}+$ neuronal cells in the right and left $\mathrm{NG}$, respectively. Fewer neurons immunoreactive to SP and FB simultaneously show the presence of nNOS and/or GAL. In the right NG, nNOS was noted in $41.83 \% \pm 2.62 \%$ of all $\mathrm{FB}+/ \mathrm{SP}+$ neurons, and in the left, nNOS was noted in $50.88 \% \pm 4.20 \%$. In the case of GAL, these values amounted to $41.31 \% \pm 3.55 \%$ and $32.70 \% \pm 3.75 \%$, respectively. The lowest percentage of SP- 
TABLE 2: The number of SP-positive neurons and their neurochemical characterization in the right and left side of the nodose ganglia. The results were considered statistically significant at $P \leq 0.05$.

\begin{tabular}{lcccr}
\hline Neuronal factor & Control & Right GN & \multicolumn{2}{c}{ Left GN } \\
\hline SP+/VIP+ & $26.88 \pm 2.94$ & $56.54 \pm 2.28^{*}$ & Control & $62.14 \pm 2.97^{*}$ \\
SP+/nNOS+ & $41.83 \pm 2.62$ & $60.81 \pm 2.38^{*}$ & $50.88 \pm 4.20$ & $65.12 \pm 2.11^{*}$ \\
SP+/CGRP+ & $53.72 \pm 2.84$ & $62.37 \pm 1.88^{*}$ & $50.78 \pm 2.92$ & $62.01 \pm 2.97^{*}$ \\
SP+/GAL+ & $41.31 \pm 3.55$ & $52.98 \pm 1.91^{*}$ & $32.70 \pm 3.75$ & $55.51 \pm 2.21^{*}$ \\
\hline
\end{tabular}

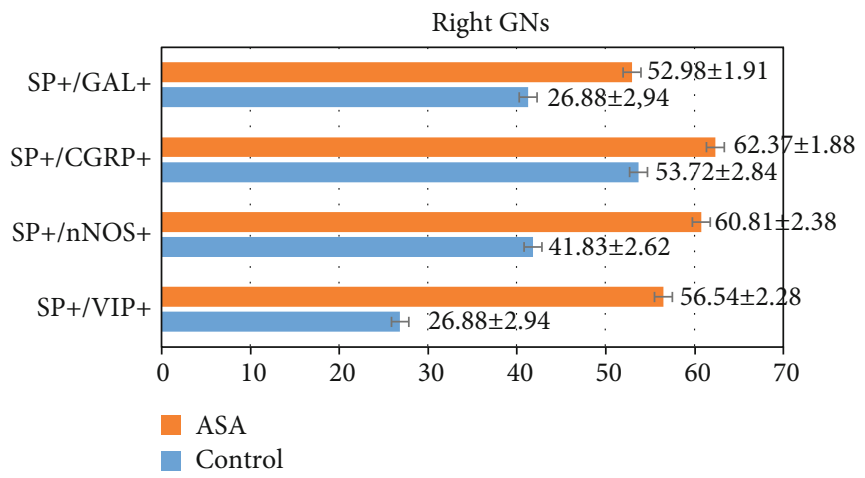

FIGURE 1: The number of SP-positive neurons and their neurochemical characterization in the right side of the nodose ganglia.

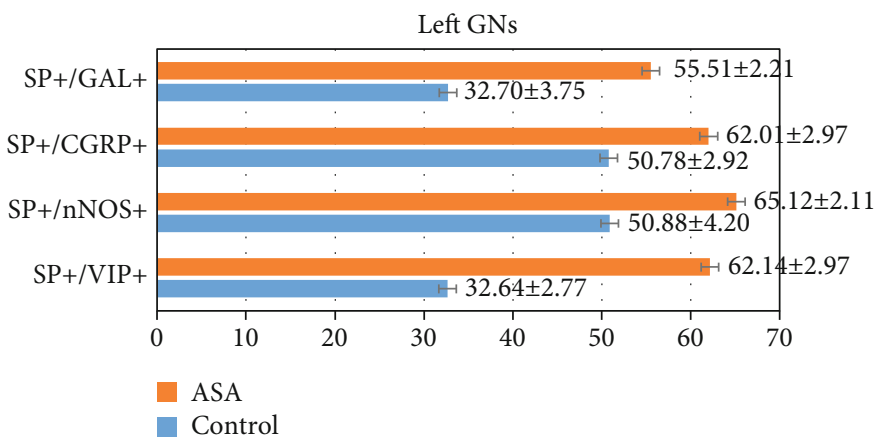

Figure 2: The number of SP-positive neurons and their neurochemical characterization in the left side of the nodose ganglia.

positive cells supplying the prepyloric region of the stomach showed immunoreactivity to VIP. FB+/SP+VIP+ neuronal cells accounted for $26.88 \% \pm 2.94 \%$ and $32.64 \% \pm 2.77 \%$ of all $\mathrm{FB}+/ \mathrm{SP}+$ neurons in the right and left $\mathrm{NG}$, respectively. Moreover, differences in the degree of colocalization of SP with other substances were noted between the right and left nodose ganglia. In the case of CGRP and/or GAL, the degree of colocalization was higher in the right NG, while a greater number of $\mathrm{FB}+/ \mathrm{SP}+$ cells in the left $\mathrm{NG}$ simultaneously showed immunoreactivity to nNOS and/or VIP.

Aspirin administration changed the degree of colocalization of SP with all the investigated substances. Generally, these changes were manifested by the increase in the percentage of neurons immunoreactive to all substances studied in relation to all $\mathrm{FB}+/ \mathrm{SP}+$ cells, but their intensity depended on the type of substance (Table 2) (Figures 1 and 2; Figures 3 and 4). The most visible changes concerned the degree of colocalization of SP and VIP. After ASA adminis- tration in the right $\mathrm{NG}$, the percentage of $\mathrm{FB}+/ \mathrm{SP}+\mathrm{VIP}+$ cells achieved $56.54 \% \pm 2.28 \%$ of all cells immunoreactive to FB and SP (an increase of about 30 percentage points (pp) in comparison to control animals), and in the left NG, the percentage amounted to $62.14 \% \pm 2.97 \%$ (an increase of above $30 \mathrm{pp}$ ). Less visible changes concerned the colocalization of SP with $\mathrm{nNOS}$ and/or CGRP. Under the influence of ASA, FB+/SP+/nNOS+ neuronal cells amounted to 60.81 $\% \pm 2.38 \%$ in the right NG (an increase of about $19 \mathrm{pp}$ ) and $65.12 \% \pm 2.11 \%$ in the left NG (an increase of nearly $15 \mathrm{pp}$ ). For CGRP, these values achieved $62.37 \% \pm 1.88 \%$ (an increase of about $9 \mathrm{pp}$ ) and $62.01 \% \pm 2.97 \%$ (an increase of about $12 \mathrm{pp}$ ), respectively. The influence of aspirin on the degree of colocalization of SP with GAL appeared interesting. In particular, ASA administration caused only a slight increase in the percentage of $\mathrm{FB}+/ \mathrm{SP}+/ \mathrm{GAL}+$ neurons in the right NG (from $41.31 \% \pm 5.55 \%$ to $52.98 \% \pm 1.91 \%$, thus by about $11 \mathrm{pp}$ ) while within the left NG, the observed 

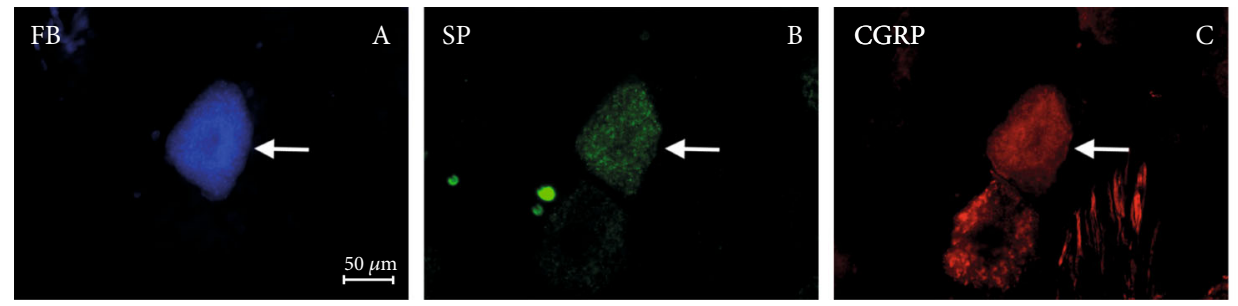

(a)
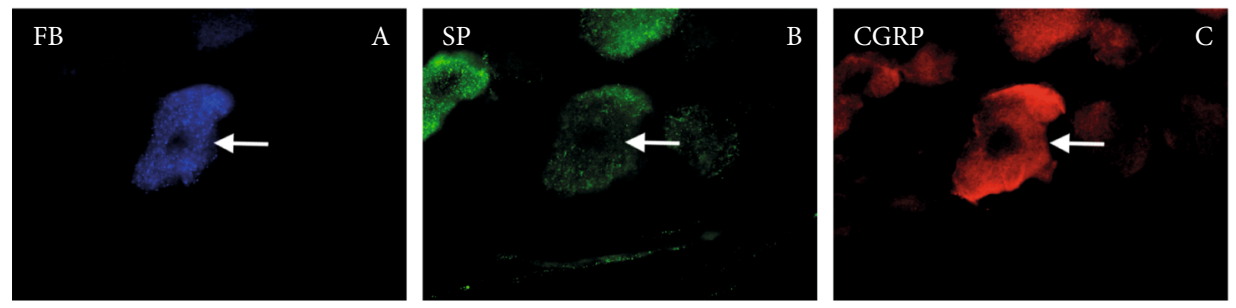

(b)
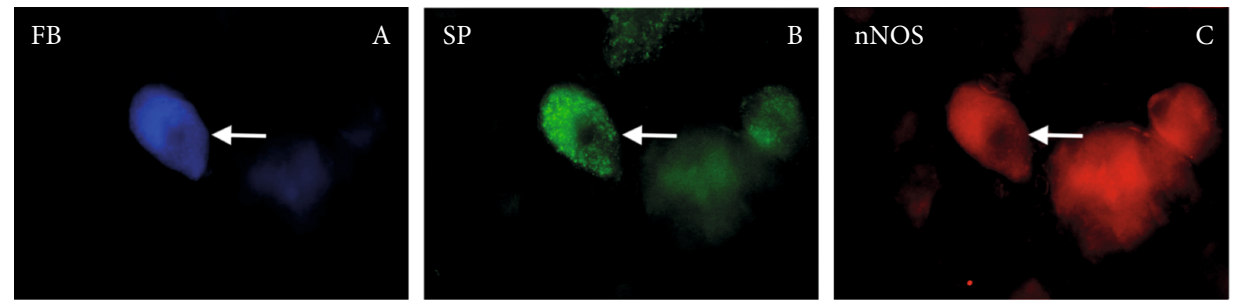

(c)
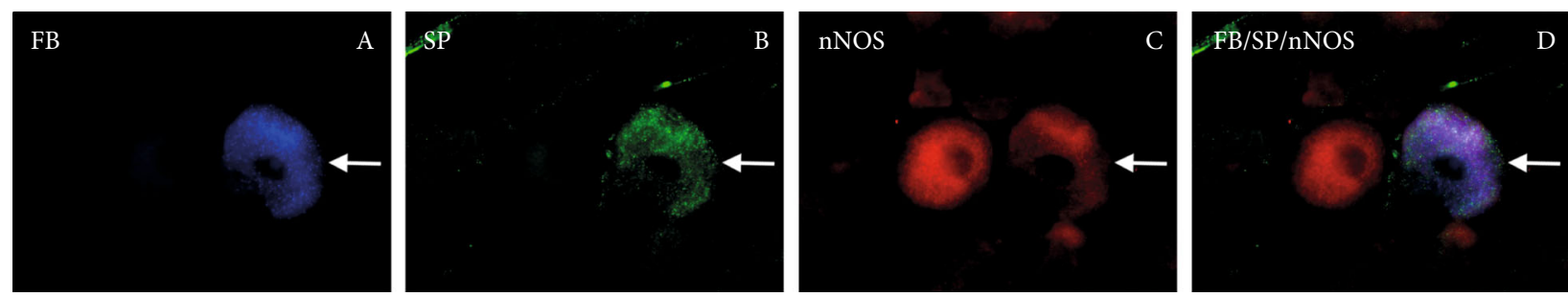

(d)
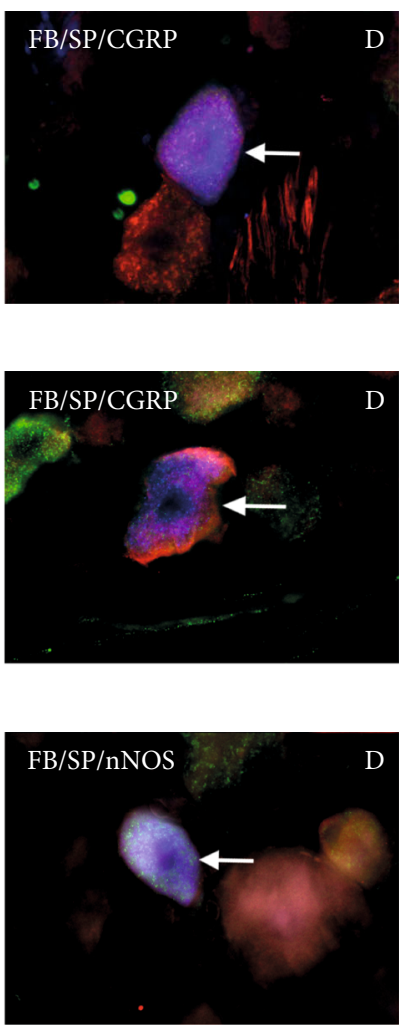

D

FIGURE 3: Fluorescence photographs showing perikarya (arrows) immunoreactive to the following: (a) FB (A), SP (B), and CGRP (C); (D) shows double labeling FB/SP/CGRP of the nodose ganglion neurons in the control group. (b) FB (A), SP (B), and CGRP (C); (D) shows double labeling FB/SP/CGRP of the nodose ganglion neurons in the ASA group. (c) FB (A), SP (B), and nNOS (C); (D) shows double labeling FB/SP/CGRP of the nodose ganglion neurons in the control group. (d) FB (A), SP (B), and nNOS (C); (D) shows double labeling $\mathrm{FB} / \mathrm{SP} / \mathrm{CGRP}$ of the nodose ganglion neurons in the ASA group.

changes were relatively significant (an increase from 32.70 $\% \pm 3.75 \%$ to $55.51 \% \pm 2.21 \%$, thus by about $23 \mathrm{pp}$ ).

Moreover, microscopic inflammatory changes, such as hyperemia, edema, and lymphatic infiltration, were demonstrated during histopathological examination of the gastric mucosal layer in animals after aspirin administration. Long-term administration of ASA triggered hyperemia and numerous erosions and ulcerations in the mucosal surfaces of the stomach. Histopathological examination performed on the wall of the gastric prepyloric area collected from animals of the ASA group confirmed gastritis caused by the ASA treatment. Microscopic changes such as superficial erosions, hyperemia, infiltration of eosinophils, and proliferation of lymphocytein in the gastric mucosa were also observed.

\section{Discussion}

Previous studies have described how neurons supplying the prepyloric area of the porcine stomach are characterized by the presence of a wide range of active substances, including (among others) SP, CGRP, GAL, VIP, and nNOS. Longterm aspirin administration caused changes in the expression of all the studied substances [11]. The results of the present investigation confirm previous studies in which SP-positive neurons supplying the stomach were described in the nodose ganglia [11, 31]. Moreover, this study found that this neuronal population is not homogeneous and demonstrates a wide range of other neuronal substances, which colocalize with substance P. Since substances occurring in the same cells often play similar functions $[11,31]$, these observations 

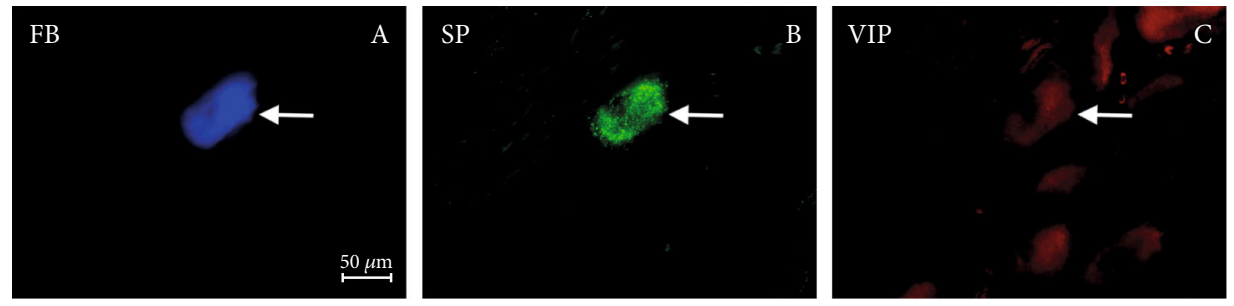

(a)
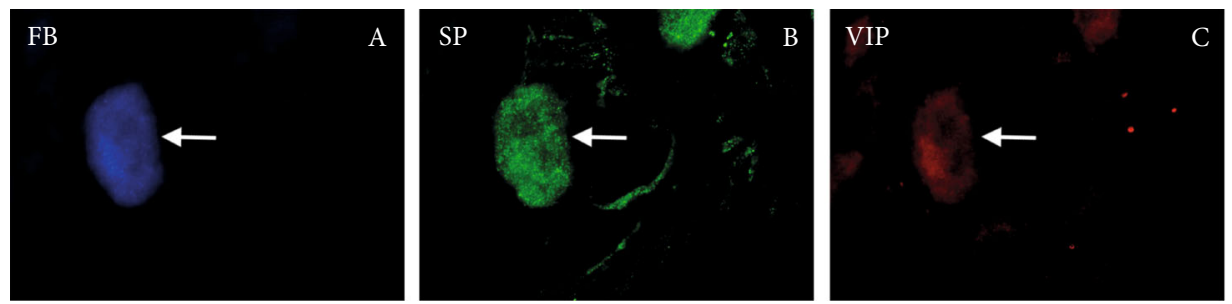

(b)
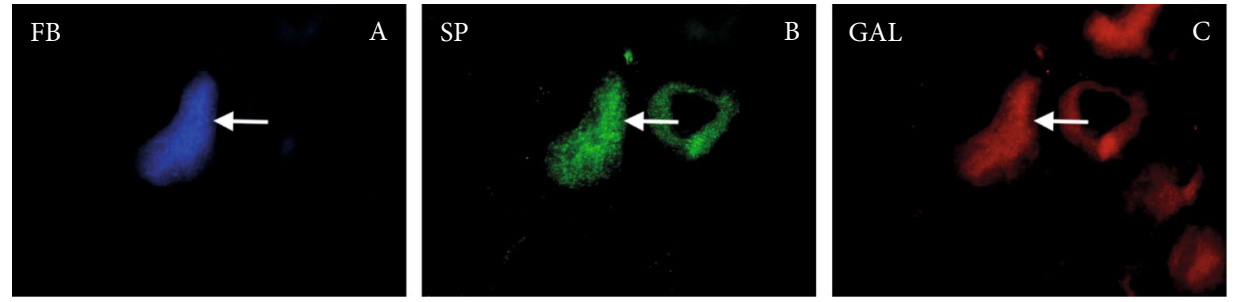

(c)
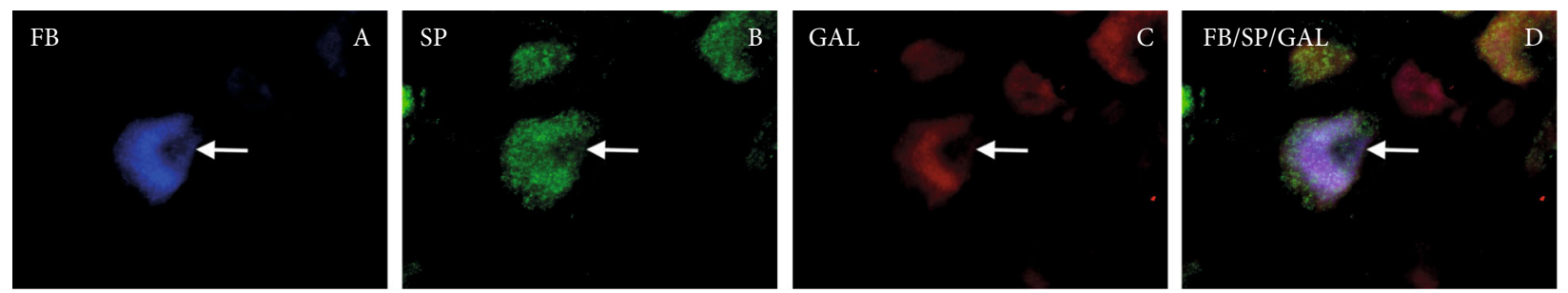

(d)

FIGURE 4: Fluorescence photographs showing perikarya (arrows) immunoreactive to the following: (a) FB (A), SP (B), and VIP (C); (D) shows double labeling FB/SP/CGRP of the nodose ganglion neurons in the control group. (b) FB (A), SP (B), and VIP (C); (D) shows double labeling FB/SP/CGRP of the nodose ganglion neurons in the ASA group. (c) FB (A), SP (B), and GAL (C); (D) shows double labeling FB/SP/CGRP of the nodose ganglion neurons in the control group. (d) FB (A), SP (B), and GAL (C); (D) shows double labeling FB/SP/CGRP of the nodose ganglion neurons in the ASA group.

may suggest that such a situation takes place in the case of SP and other substances studied during the present investigations. In control animals, the highest degree of colocalization concerned SP and CGRP. These two substances have been noted in the same neuronal cells within different parts of the central and peripheral nervous system, including (among others) the brain, dorsal root ganglia, enteric neurons, and prevertebral ganglia. This is not unusual, because CGRP (like $\mathrm{SP}$ ) is known as one of the most important factors involved in sensory and/or pain stimulus conduction [32]. Moreover, both SP and CGRP in the digestive system may modulate the gastrointestinal secretory activity [33], blood flow in the wall of the digestive tract [34], and intestinal motility (although CGRP is not a typical factor regulating smooth muscle activity) [35].

Other substances observed during the present study in SP-positive neurons supplying the stomach may also show activities similar to actions of substance P. Namely, GAL takes part in the regulation of intestinal motility. The influence of GAL on the intestinal muscles may result in stimulatory or inhibitory effects depending on animal species and the intestinal fragment [36-38]. Similar situations have been described in the case of SP $[39,40]$. Moreover, GAL (just like $\mathrm{SP}$ ) is involved in regulatory processes connected with gastrointestinal secretory activity [41]. In turn, VIP and nitric oxide (in the present study, nNOS was used as a marker of nitrergic neurons) are known as the most important inhibitory factors within the gastrointestinal tract [42]. They influence the intestinal muscles causing relaxatory effects [42], reduce the secretory activity of the digestive tract [43], and regulate the mesenteric blood flow [44]. Moreover, the majority of substances studied in the present investigation are involved in immunological processes and show neuroprotective activity $[45,46]$. It should be pointed out that the 
participation of SP and VIP in immunological processes shows an opposite character. Namely, SP is known as a potent proinflammatory factor [47], while VIP demonstrates anti-inflammatory activity [48]. The cooperation of the above-mentioned substances located in the same neurons in this respect remains unclear.

The obtained results show that aspirin administration may affect the neurochemical coding of neurons within the NG supplying the stomach, which is in agreement with previous studies [49]. Changes in neurochemical characterization of SP-positive neuronal cells may be connected with various mechanisms. The most commonly observed fluctuations are the result of the irritant effects of ASA on the gastric mucosal layer and/or inflammatory processes induced by this drug. Such activities of aspirin are relatively well known and have been described in previous studies [50]. Moreover, this inflammatory process has also been confirmed in the gastric mucosal layer during the present investigation. The possibility that the observed changes are the result of inflammation is supported by the fact that the majority of the studied substances take part in immunological processes and may affect the levels of pro- or anti-inflammatory factors [47, 48, 51, 52]. On the other hand, fluctuations in the chemical coding of SP-positive neurons may arise from the influence of inflammation on the conduction of sensory and pain stimuli. It is more likely that SP and CGRP are factors which are involved in sensory innervation [53] and nodose ganglia are typical sensory ganglia [54]. Of course, the other reasons for the observed changes cannot be excluded. They may be the result of the direct influence of ASA on sensory nerve endings in the gastric mucosal layer and manifestation of adaptive neuroprotective and/or reparative processes. This view is supported by two facts. Firstly, it is known that aspirin may affect the nervous system [49]. Secondly, the majority of substances studied in this investigation are involved in neuroprotective processes and neurogenesis after nerve damage during various pathological processes $[45,55]$. Moreover, it is well established that the expression of factors involved in neuroprotective reactions usually increases under pathological factors $[49,55]$ and such changes were also noted during the present study. The reasons for the observed changes are also unclear. These changes could be the result of the inhibition of active substances being transported from the cell body to neuronal endings and synapses [56]. On the other hand, they may also be connected with fluctuations in neuropeptide synthesis, which may concern various stages of this process, such as transcription, translation, and posttranslational and/or metabolic disturbances [57].

In conclusion, the obtained results show that SP-positive neurons located in NG and supplying the stomach are differentiated according to the occurrence of other active substances, which likely function as comediators. Moreover, aspirin administration affects the neurochemical coding of these neurons. Changes observed under ASA action generally rely on the increase in the degree of colocalization of SP with other substances and are probably connected with inflammatory processes and/or neuroprotective activity. Nevertheless, due to the multidirectional activity of the neu- ronal substances studied and various pathological mechanisms connected with aspirin-induced changes, several aspects concerning the functioning of SP-LI neurons located in NG and innervating the stomach both under physiological conditions and during pathological processes remain unclear and require further studies.

\section{Abbreviations \\ SP: $\quad$ Substance P \\ NG: $\quad$ Nodose ganglia \\ FB: $\quad$ Fast Blue \\ CGRP: Calcitonin gene-related peptide \\ GAL: Galanin \\ nNOS: Neuronal isoform of nitric oxide synthase \\ VIP: Vasoactive intestinal polypeptide.}

\section{Data Availability}

The data used to support the findings of this study are available from the corresponding author upon request.

\section{Additional Points}

Key Points. (1) Although the mechanisms of aspirin activity have been the subject of many studies, the knowledge concerning its influence on gastrointestinal innervation is extremely scanty. (2) Aspirin causes changes in all the investigated substances, and the most visible changes concerned the number of neurons immunoreactive to SP and VIP. (3) Aspirin shows effects which are particularly evident within the stomach, where taking this drug may result in the gastric mucosal injury.

\section{Conflicts of Interest}

The authors declare that they have no conflicts of interest.

\section{Acknowledgments}

This publication was funded by the KNOW (Leading National Research Centre) Scientific Consortium "Healthy Animal-Safe Food", decision of the Ministry of Science and Higher Education (No. 05-1/KNOW2/2015). The project was financially cosupported by the Ministry of Science and Higher Education in the range of the program entitled "Regional Initiative of Excellence" for the years 2019-2022 (Project No. 010/RID/2018/19, amount of funding 12,000,000 PLN).

\section{References}

[1] J. B. Furness, B. P. Callaghan, L. R. Rivera, and H. J. Cho, "The enteric nervous system and gastrointestinal innervation: integrated local and central control," Advances in Experimental Medicine and Biology, vol. 817, pp. 39-71, 2014.

[2] C. Piasecki, "Observations on the submucous plexus and mucosal arteries of the dog's stomach and first part of the duodenum," Journal of Anatomy, vol. 119, Part 1, pp. 133-148, 1975. 
[3] I. Lolova, N. Lolov, and M. Papasova, "Structure of the myenteric plexus in the sphincters of cat gastro-intestinal tract. III. Character and distribution of the dense-core vesicles in the axonal varicosities," Acta Physiologica et Pharmacologica Bulgarica, vol. 6, no. 1, pp. 76-84, 1980.

[4] R. J. Phillips and T. L. Powley, "Macrophages associated with the intrinsic and extrinsic autonomic innervation of the rat gastrointestinal tract," Autonomic Neuroscience, vol. 169, no. 1, pp. 12-27, 2012.

[5] I. Kasacka, Z. Piotrowska, H. Car, I. Janiuk, and W. Lebkowski, "Cocaine- and amphetamine-regulated transcript: identification and distribution in human gastrointestinal tract," Journal of Biological Regulators and Homeostatic Agents, vol. 26, no. 3, pp. 419-428, 2012.

[6] N. Bódi, I. Battonyai, P. Talapka, E. Fekete, and M. Bagyánszki, "Spatial pattern analysis of nitrergic neurons in the myenteric plexus of the duodenum of different mammalian species," Acta Biologica Hungarica, vol. 60, no. 4, pp. 347-358, 2009.

[7] J. B. Furness, J. C. Bornstein, and T. K. Smith, "The normal structure of gastrointestinal innervation," Journal of Gastroenterology and Hepatology, vol. 5, no. S-1, pp. 1-9, 1990.

[8] T. Hayakawa, S. Kuwahara-Otani, S. Maeda, K. Tanaka, and M. Seki, "Brain-derived neurotrophic factor immunoreactive vagal sensory neurons innervating the gastrointestinal tract of the rat," Journal of Chemical Neuroanatomy, vol. 61-62, pp. 83-87, 2014.

[9] F. Ochoa-Cortes, R. Guerrero-Alba, E. E. Valdez-Morales et al., "Chronic stress mediators act synergistically on colonic nociceptive mouse dorsal root ganglia neurons to increase excitability," Neurogastroenterology and Motility, vol. 26, no. 3, pp. 334-345, 2014.

[10] J. P. Miolan and J. P. Niel, “The mammalian sympathetic prevertebral ganglia: integrative properties and role in the nervous control of digestive tract motility," Journal of the Autonomic Nervous System, vol. 58, no. 3, pp. 125-138, 1996.

[11] L. Rytel and J. Całka, "Acetylsalicylic acid-induced changes in the chemical coding of extrinsic sensory neurons supplying the prepyloric area of the porcine stomach," Neuroscience Letters, vol. 617, pp. 218-224, 2016.

[12] K. Palus and J. Całka, "Neurochemical plasticity of the coeliacsuperior mesenteric ganglion complex neurons projecting to the prepyloric area of the porcine stomach following hyperacidity," Neural Plasticity, vol. 2016, 9 pages, 2016.

[13] S. Gonkowski, "Substance P as a neuronal factor in the enteric nervous system of the porcine descending colon in physiological conditions and during selected pathogenic processes," BioFactors, vol. 39, no. 5, pp. 542-551, 2013.

[14] P. W. Mantyh, "Neurobiology of substance P and the NK1 receptor," The Journal of Clinical Psychiatry, vol. 11, pp. 6-10, 2002.

[15] T. Hökfelt, B. Pernow, and J. Wahren, "Substance P: a pioneer amongst neuropeptides," Journal of Internal Medicine, vol. 249, no. 1, pp. 27-40, 2001.

[16] P. Holzer, "Stimulation and inhibition of gastrointestinal propulsion induced by substance $\mathrm{P}$ and substance $\mathrm{K}$ in the rat," British Journal of Pharmacology, vol. 86, no. 1, pp. 305-312, 1985.

[17] T. S. Gates, R. P. Zimmerman, C. R. Mantyh et al., "Substance $\mathrm{P}$ and substance $\mathrm{K}$ receptor binding sites in the human gastrointestinal tract: localization by autoradiography," Peptides, vol. 9, no. 6, pp. 1207-1219, 1988.
[18] M. El-Salhy and A. Spångéus, "Substance P in the gastrointestinal tract of non-obese diabetic mice," Scandinavian Journal of Gastroenterology, vol. 33, no. 4, pp. 394-400, 1998.

[19] I. Karagiannides and C. Pothoulakis, "Substance P, obesity, and gut inflammation," Current Opinion in Endocrinology, Diabetes, and Obesity, vol. 16, no. 1, pp. 47-52, 2009.

[20] L. Barthó and P. Holzer, "Search for a physiological role of substance $\mathrm{P}$ in gastrointestinal motility," Neuroscience, vol. 16, no. 1, pp. 1-32, 1985.

[21] J. Goldhill, M. F. Porquet, and N. Selve, "Antisecretory and relaxatory effects of tachykinin antagonists in the guinea-pig intestinal tract," The Journal of Pharmacy and Pharmacology, vol. 51, no. 9, pp. 1041-1048, 1999.

[22] E. K. Reinke, M. J. Johnson, C. Ling et al., "Substance P receptor mediated maintenance of chronic inflammation in EAE," Journal of Neuroimmunology, vol. 180, no. 1-2, pp. 117-125, 2006.

[23] J. Zhu, C. Qu, X. Lu, and S. Zhang, "Activation of microglia by histamine and substance P," Cellular Physiology and Biochemistry, vol. 34, no. 3, pp. 768-780, 2014.

[24] W. W. Pawlik, P. Gustaw, K. Czarnobilski, R. Sendur, and S. J. Konturek, "Effects of substance $\mathrm{P}$ on intestinal circulation and oxygen consumption," Acta Physiologica Polonica, vol. 38, no. 5, pp. 410-417, 1987.

[25] N. Y. Lai, K. Mills, and I. M. Chiu, "Sensory neuron regulation of gastrointestinal inflammation and bacterial host defence," Journal of Internal Medicine, vol. 282, no. 1, pp. 5-23, 2017.

[26] J. Peng and Y. J. Li, "The vanilloid receptor TRPV1: role in cardiovascular and gastrointestinal protection," European Journal of Pharmacology, vol. 627, no. 1-3, pp. 1-7, 2010.

[27] B. L. Fiebich, K. Lieb, N. Kammerer, and M. Hüll, "Synergistic inhibitory effect of ascorbic acid and acetylsalicylic acid on prostaglandin E2 release in primary rat microglia," Journal of Neurochemistry, vol. 86, pp. 173-178, 2004.

[28] M. Tanaka, A. Tanaka, K. Suemaru, and H. Araki, "The assessment of risk for gastrointestinal injury with anticoagulant and antiplatelet drugs: the possible beneficial effect of eicosapentaenoic acid for the risk of gastrointestinal injury," Biological \& Pharmaceutical Bulletin, vol. 36, no. 2, pp. 222 227, 2013.

[29] S. C. Ng and F. K. L. Chan, "NSAID-induced gastrointestinal and cardiovascular injury," Current Opinion in Gastroenterology, vol. 26, no. 6, pp. 611-617, 2010.

[30] A. S. Tarnawski, A. Ahluwalia, and M. K. Jones, "Increased susceptibility of aging gastric mucosa to injury: the mechanisms and clinical implications," World Journal of Gastroenterology, vol. 20, no. 16, pp. 4467-4482, 2014.

[31] M. Zalecki, "Extrinsic primary afferent neurons projecting to the pylorus in the domestic pig-localization and neurochemical characteristics," Journal of Molecular Neuroscience, vol. 52, no. 1, pp. 82-89, 2014.

[32] H. Matsunami, "CGRP $\alpha$-expressing sensory neurons respond to stimuli that evoke sensations of pain and itch," PLoS One, vol. 7, no. 5, article e36355, 2012.

[33] P. Holzer, "Implications of tachykinins and calcitonin generelated peptide in inflammatory bowel disease," Digestion, vol. 59, no. 4, pp. 269-283, 1998.

[34] R. Uddman, L. Edvinsson, E. Ekblad, R. Håkanson, and F. Sundler, "Calcitonin gene-related peptide (CGRP): perivascular distribution and vasodilatory effects," Regulatory Peptides, vol. 15, no. 1, pp. 1-23, 1986. 
[35] I. E. Demir, K. H. Schäfer, E. Tieftrunk, H. Friess, and G. O. Ceyhan, "Neural plasticity in the gastrointestinal tract: chronic inflammation, neurotrophic signals, and hypersensitivity," Acta Neuropathologica, vol. 125, no. 4, pp. 491-509, 2013.

[36] R. P. Korolkiewicz, Z. Konstański, P. Rekowski et al., "Sources of activator $\mathrm{Ca} 2+$ for galanin-induced contractions of rat gastric fundus, jejunum and colon," Journal of Physiology and Pharmacology, vol. 51, pp. 821-831, 2000.

[37] A. Zacharko-Siembida, J. L. Valverde Piedra, S. Szymańczyk, and M. B. Arciszewski, "Immunolocalization of NOS, VIP, galanin and SP in the small intestine of suckling pigs treated with red kidney bean (Phaseolus vulgaris) lectin," Acta Histochemica, vol. 115, no. 3, pp. 219-225, 2013.

[38] A. Czujkowska and M. B. Arciszewski, "Galanin is coexpressed with substance $\mathrm{P}$, calbindin and corticotropinreleasing factor (CRF) in the enteric nervous system of the wild boar (Sus scrofa) small intestine," Anatomia, Histologia, Embryologia, vol. 45, no. 2, pp. 115-123, 2016.

[39] J. R. Grider, "Regulation of excitatory neural input to longitudinal intestinal muscle by myenteric interneurons," The American Journal of Physiology, vol. 275, no. 5, pp. G973-G978, 1998.

[40] K. Nieber, P. Oehme, and K. Milenov, "Different action of substance P on gastric and ileal smooth muscle," Die Pharmazie, vol. 37, no. 9, pp. 656-658, 1982.

[41] G. Soldani, G. Mengozzi, A. Della Longa, L. Intorre, F. Martelli, and D. R. Brown, "An analysis of the effects of galanin on gastric acid secretion and plasma levels of gastrin in the dog," European Journal of Pharmacology, vol. 154, no. 3, pp. 313-318, 1988.

[42] L. A. Van Geldre and R. A. Lefebvre, "Interaction of NO and VIP in gastrointestinal smooth muscle relaxation," Current Pharmaceutical Design, vol. 10, no. 20, pp. 2483-2497, 2004.

[43] M. J. Farthing, "Antisecretory drugs for diarrheal disease," Digestive Diseases, vol. 24, no. 1-2, pp. 47-58, 2006.

[44] C. Maake, C. Kaufmann, and M. Reinecke, "Ontogeny of neurohormonal peptides, serotonin, and nitric oxide synthase in the gastrointestinal neuroendocrine system of the axolotl (Ambystoma mexicanum): an immunohistochemical analysis," General and Comparative Endocrinology, vol. 121, no. 1, pp. 74-83, 2001.

[45] K. Palus and J. Całka, "Alterations of neurochemical expression of the coeliac-superior mesenteric ganglion complex (CSMG) neurons supplying the prepyloric region of the porcine stomach following partial stomach resection," Journal of Chemical Neuroanatomy, vol. 72, pp. 25-33, 2016.

[46] D. E. Barañano and S. H. Snyder, "Neural roles for heme oxygenase: contrasts to nitric oxide synthase," Proceedings of the National Academy of Sciences of the United States of America, vol. 98, no. 20, pp. 10996-11002, 2001.

[47] E. D. de Avila, R. S. de Molon, D. A. de Godoi Gonçalves, and C. M. Camparis, "Relationship between levels of neuropeptide substance $\mathrm{P}$ in periodontal disease and chronic pain: a literature review," Journal of Investigative and Clinical Dentistry, vol. 5, no. 2, pp. 91-97, 2014.

[48] P. Kong, R. Wu, X. Liu et al., "The effects of anti-inflammatory drug treatment in gastric cancer prevention: an update of a meta-analysis," Journal of Cancer, vol. 7, no. 15, pp. 22472257, 2016.
[49] K. Palus and J. Całka, "The influence of prolonged acetylsalicylic acid supplementation-induced gastritis on the neurochemistry of the sympathetic neurons supplying prepyloric region of the porcine stomach," PLoS One, vol. 10, no. 11, article e0143661, 2015.

[50] T. Thongbai, "The prevalence of gastroduodenal mucosal injuries in aspirin users," Journal of the Medical Association of Thailand, vol. 96, pp. 1423-1427, 2013.

[51] L. Edvinsson, "CGRP receptor antagonists and antibodies against CGRP and its receptor in migraine treatment," British Journal of Clinical Pharmacology, vol. 80, no. 2, pp. 193-199, 2015.

[52] R. Lang, A. L. Gundlach, F. E. Holmes et al., "Physiology, signaling, and pharmacology of galanin peptides and receptors: three decades of emerging diversity," Pharmacological Reviews, vol. 67, pp. 118-175, 2014.

[53] G. R. Kestell, R. L. Anderson, J. N. Clarke, R. V. Haberberger, and I. L. Gibbins, "Primary afferent neurons containing calcitonin gene-related peptide but not substance $\mathrm{P}$ in forepaw skin, dorsal root ganglia, and spinal cord of mice," The Journal of Comparative Neurology, vol. 523, no. 17, pp. 2555-2569, 2015.

[54] R. L. Young, A. J. Page, N. J. Cooper, C. L. Frisby, and L. A. Blackshaw, "Sensory and motor innervation of the crural diaphragm by the vagus nerves," Gastroenterology, vol. 138, no. 3, pp. 1091-1101.e5, 2010.

[55] L. Rytel and J. Całka, "Neuropeptide profile changes in sensory neurones after partial prepyloric resection in pigs," Annals of Anatomy, vol. 206, pp. 48-56, 2016.

[56] M. M. Santafé, N. Garcia, M. A. Lanuza, and J. Tomàs, "Protein kinase $\mathrm{C}$ activity affects neurotransmitter release at polyinnervated neuromuscular synapses," Journal of Neuroscience Research, vol. 85, no. 7, pp. 1449-1457, 2007.

[57] H. Onken, S. B. Moffett, and D. F. Moffett, "The anterior stomach of larval mosquitoes (Aedes aegypti): effects of neuropeptides on transepithelial ion transport and muscular motility," The Journal of Experimental Biology, vol. 207, no. 21, pp. 3731-3739, 2004. 